\title{
ON THE BOUNDED LAWS OF ITERATED LOGARITHM IN BANACH SPACE
}

\author{
Dianliang Deng ${ }^{1}$
}

\begin{abstract}
In the present paper, by using the inequality due to Talagrand's isoperimetric method, several versions of the bounded law of iterated logarithm for a sequence of independent Banach space valued random variables are developed and the upper limits for the non-random constant are given.
\end{abstract}

Mathematics Subject Classification. 60F05, 60B12, 60F99.

Received March 26, 2004.

\section{IntRoduction}

Let $B$ be a real separable Banach space with $\|\cdot\|$ and topological dual $B^{*}$. For a $B$-valued random variable $X$ and some $p \in[1,2]$, we write $X \in W M_{0}^{p}$ if for all $f \in B^{*}$, we have $E f(X)=0$ and $E|f(X)|^{p}<\infty$. Throughout $\left\{X_{n}, n \geq 1\right\}$ is a sequence of independent $B$-valued random variables defined on a probability space $(\Omega, F, P)$ and $\left\{\epsilon_{n}, n \geq 1\right\}$ is an independent Rademacher series supported on the same probability space $(\Omega, F, P)$ and independent of $\left\{X_{n}, n \geq 1\right\}$. For each $n \geq 1$, put $S_{n}=\sum_{i=1}^{n} X_{i}, W_{n, p}=\sup _{f \in B_{1}^{*}}\left(\sum_{i=1}^{n}\left|f\left(X_{i}\right)\right|^{p}\right)^{1 / p}, s_{n, p}=$ $\sup _{f \in B_{1}^{*}}\left(\sum_{i=1}^{n} E\left|f\left(X_{i}\right)\right|^{p}\right)^{1 / p}$ where $B_{1}^{*}$ is the unit ball of $B^{*}$. As usual, $L_{2} x$ denotes the function $\log \log \max \left\{e^{e}, x\right\}$.

Griffin and Kuelbs [10,11] established some extensions of the law of iterated logarithm (LIL) via selfnormalizations for independent real valued random variables both in the symmetric and non-symmetric cases. However, many of these results require a symmetry assumption. Afterwards, Godbole [9] discussed the selfnormalized bounded law of iterated logarithm (SNBLIL) for $B$-valued random variables and gave the following definition for SNBLIL: $\left\{X_{n}, n \geq 1\right\}$ will be said to satisfy the SNBLIL $\left(\left\{X_{n}\right\} \in S N B L I L\right)$ if there exists a non-random constant $0<M<\infty$ such that for some $p \in[1,2]$ and $r>0$,

$$
\limsup _{n \rightarrow \infty} \frac{\left\|\sum_{i=1}^{n} X_{i}\right\|}{\left(\sum_{i=1}^{n}\left\|X_{i}\right\|^{p}\right)^{1 / p}\left(L_{2} \sum_{i=1}^{n}\left\|X_{i}\right\|^{p}\right)^{r}}=M \quad \text { a.s. }
$$

Godbole [9] proved that under some conditions, $\left\{X_{n}, n \geq 1\right\} \in S N B L I L$ for $r=\frac{1}{2}$ and $r=\frac{p-1}{p}$. The advantage of SNBLIL is to drop the standard bounded assumption for the random variables $\left\{X_{n}, n \geq 1\right\}$. But these theorems do not give the accurate value for the non-random constant $M$ and deeply depend on the type of Banach spaces. In the case of real symmetric random variables, Marcinkiewicz proved that $M \leq 1$ for $p=2$

\footnotetext{
Keywords and phrases. Banach space, bounded law of iterated logarithm, isoperimetric inequality, rademacher series, selfnormalizer.

1 Department of Mathematics and Statistics, University of Regina, 3737 Wascana Parkway, Regina, SK, S4S 0A2 Canada; deng@math.uregina.ca
}

(C) EDP Sciences, SMAI 2005 
and $r=\frac{1}{2}$ (see [11] for a simple proof of Marcinkiewicz's result). Therefore it was of interest to ask whether $M \leq 1$ for $B$-valued symmetric random variables or to give the accurate estimate for $M$. By replacing the self-normalizer $\left(\sum_{i=1}^{n}\left\|X_{i}\right\|^{p}\right)^{1 / p}\left(L_{2} \sum_{i=1}^{n}\left\|X_{i}\right\|^{p}\right)^{r}$ by $\left(\sup _{f \in B_{1}^{*}} \sum_{i=1}^{n}\left|f\left(X_{i}\right)\right|^{p}\right)^{1 / p}\left(L_{2} \sup _{f \in B_{1}^{*}} \sum_{i=1}^{n}\left|f\left(X_{i}\right)\right|^{p}\right)^{r}$, Deng [6] proved the following results.

Theorem 1.1. Let $\left\{X_{n}, n \geq 1\right\}$ be a sequence of independent symmetric B-valued random variables. Suppose that for some $p \in[1,2]$, the following conditions hold:

$$
\begin{gathered}
\lim _{n \rightarrow \infty} W_{n, p}^{p} \rightarrow+\infty \text { a.s. } \\
\frac{\sup _{f \in D}\left|f\left(X_{n}\right)\right|\left(L_{2} W_{n, p}^{p}\right)^{1 / 2}}{W_{n, p}} \rightarrow 0 \text { a.s. } \\
S_{n} /\left(2 W_{n, p}^{2} L_{2} W_{n, p}^{p}\right)^{1 / 2} \rightarrow 0 \text { in probability. }
\end{gathered}
$$

Then

$$
\limsup _{n \rightarrow \infty} \frac{\left\|S_{n}\right\|}{\left(2 W_{n, p}^{2} L_{2} W_{n, p}^{p}\right)^{1 / 2}} \leq 1 \text { a.s. }
$$

In particular, if (1.2), (1.3) and (1.4) hold for $p=2$, then

$$
\limsup _{n \rightarrow \infty} \frac{\left\|S_{n}\right\|}{\left(2 W_{n, 2}^{2} L_{2} W_{n, 2}^{2}\right)^{1 / 2}}=1 \text { a.s. }
$$

Theorem 1.2. Let $\left\{X_{n}, n \geq 1\right\}$ be a sequence of independent symmetric B-valued random variables. Suppose that for some $p \in[1,2]$, the following conditions hold:

$$
\begin{gathered}
\lim _{n \rightarrow \infty} W_{n, p}^{p}=+\infty \text { a.s. } \\
\frac{\sup _{f \in D}\left|f\left(X_{n}\right)\right|\left(L_{2} W_{n, p}^{p}\right)^{\frac{p-1}{p}}}{W_{n, p}} \rightarrow 0 \text { a.s. } \\
S_{n} / W_{n, p}\left(L_{2} W_{n, p}^{p}\right)^{\frac{p-1}{p}} \rightarrow 0 \text { in probability. }
\end{gathered}
$$

Then

$$
\limsup _{n \rightarrow \infty} \frac{\left\|S_{n}\right\|}{2 W_{n, p}\left(L_{2} W_{n, p}^{p}\right)^{\frac{p-1}{p}}} \leq \begin{cases}\frac{1}{2} & p=1 \\ \left(\frac{p}{p-1}\right)^{\frac{p-1}{p}} & 1<p<2 \quad \text { a.s. } \\ \frac{\sqrt{2}}{2} & p=2 .\end{cases}
$$

Now it is of concern that the equation (1.1) holds if the self-normalizers $\left(\sum_{i=1}^{n}\left\|X_{i}\right\|^{p}\right)^{1 / p}\left(L_{2} \sum_{i=1}^{n}\left\|X_{i}\right\|^{p}\right)^{r}$ and $\quad\left(\sum_{i=1}^{n}\left|f\left(X_{i}\right)\right|^{p}\right)^{1 / p}\left(L_{2} \sum_{i=1}^{n}\left|f\left(X_{i}\right)\right|^{p}\right)^{r} \quad$ are replaced by the nonrandom normalizers $\left(\sum_{i=1}^{n} E\left\|X_{i}\right\|^{p}\right)^{1 / p}\left(L_{2} \sum_{i=1}^{n} E\left\|X_{i}\right\|^{p}\right)^{r}$ and $\left(\sum_{i=1}^{n} E\left|f\left(X_{i}\right)\right|^{p}\right)^{1 / p}\left(L_{2} \sum_{i=1}^{n} E\left|f\left(X_{i}\right)\right|^{p}\right)^{r}$. Moreover, if (1.1) holds for non-random normalizers, what the nonrandom constant $M$ equals? or can the estimate of upper limit for $M$ be given?

The purpose of the present paper is to solve the above questions. In some sense, we generalize the bounded law of iterated logarithm for $B$-valued random variables. In fact, the classical bounded law of iterated logarithm can be obtained for $p=2$. Our approach is mainly due to Ledoux and Talagrand [13]. In Ledoux and Talagrand [13], 
they developed the isoperimetric inequality and the finite dimensional approximation argument via some entropy estimate which is based on the Sudakov type minoration. This approach is very effective for dealing with the questions of strong limit theorems of $B$-valued random variables and plays an important role in the accurate estimates of upper limits of BLIL in Banach space. The paper is organized as follows. In Section 2, we state the main results. In Section 3, we develop a new inequality. The proofs of theorems are obtained by using an entropy approach and the new inequality.

\section{MAIN RESUltS}

We start with introducing some notations. For a sequence of independent $B$-valued random variables $\left\{X_{n}, n \geq 1\right\}$, put $S_{n}=\sum_{i=1}^{n} X_{i}, s_{n, p}=\left(\sup _{f \in B_{1}^{*}} \sum_{i=1}^{n} E\left|f\left(X_{i}\right)\right|^{p}\right)^{1 / p}$ for some $p \in[1,2]$, where $B_{1}^{*}$ is the unit ball of $B^{*}$. Note that if $D$ is a countable subset of the unit ball $B_{1}^{*}$ such that $\|x\|=\sup _{f \in D}|f(x)|$ for every $x \in B, s_{n, p}=\left(\sup _{f \in D} \sum_{i=1}^{n} E\left|f\left(X_{i}\right)\right|^{p}\right)^{1 / p}$. By replacing the self-normalizer with $s_{n, p}\left(L_{2} s_{n, p}^{p}\right)^{r}$, we may obtain the results with respect to the BLIL. At first we discuss the case that $r=\frac{1}{2}$ and state our main theorems.

Theorem 2.1. Let $\left\{X_{n}, n \geq 1\right\}$ be a sequence of independent B-valued random variables with $X_{n} \in W M_{0}^{p}$ for some $p \in[1,2]$. Assuming that the following statements hold:

$$
\begin{gathered}
\lim _{n \rightarrow \infty} s_{n, p}^{p}=+\infty \\
\left\|X_{n}\right\| \leq\left(\eta_{n} s_{n, p}\right) /\left(L_{2} s_{n, p}^{p}\right)^{1 / 2} \text { a.s. }
\end{gathered}
$$

for some sequence of real number $\left\{\eta_{n}\right\}$ with $1 \geq \eta_{n} \rightarrow 0$, and

$$
S_{n} /\left(2 s_{n, p}^{2} L_{2} s_{n, p}^{p}\right)^{1 / 2} \rightarrow 0 \text { in probability }
$$

Then

$$
\limsup _{n \rightarrow \infty} \frac{\left\|S_{n}\right\|}{\left(2 s_{n, p}^{2} L_{2} s_{n, p}^{p}\right)^{1 / 2}} \leq\left\{\begin{array}{ll}
2^{1+1 / p} & \text { for } 1 \leq p<2 \\
1 & \text { for } p=2 .
\end{array}\right. \text { a.s. }
$$

Note that the Wittmann's LIL holds under the condition that $\sum_{n=1}^{\infty} \frac{E\left\|X_{n}\right\|^{r}}{\left(s_{n}^{2} L_{2} s_{n}^{2}\right)^{r / 2}}<+\infty$ for $r>2$ where $s_{n}^{2}=s_{n, 2}^{2}=\sup _{f \in B_{1}^{*}} \sum_{i=1}^{n} E f^{2}\left(X_{i}\right)$ (see [3], Th. 2.1). Now one would like to know whether there is a similar result if $s_{n, 2}$ is replaced by $s_{n, p}$ for $1 \leq p \leq 2$. Actually we have the following result.

Theorem 2.2. Let $\left\{X_{n}, n \geq 1\right\}$ be a sequence of independent B-valued random variables with $X_{n} \in W M_{0}^{p}$ for some $p \in[1,2]$. If the following statements hold:

$$
\begin{gathered}
\sum_{n=1}^{\infty}\left(s_{n, p}^{2} L_{2} s_{n, p}^{p}\right)^{-\alpha / 2} E\left\|X_{n}\right\|^{\alpha}<+\infty \quad \text { for some } \alpha>p ; \\
s_{n, p} \rightarrow \infty .
\end{gathered}
$$

Then

$$
\limsup _{n \rightarrow \infty} \frac{\left\|S_{n}\right\|}{\left(2 s_{n, p}^{2} L_{2} s_{n, p}^{p}\right)^{1 / 2}} \leq \Gamma \quad \text { a.s. for some } \Gamma>0
$$

holds provided that $S_{n} /\left(2 s_{n, p}^{2} L_{2} s_{n, p}^{p}\right)^{1 / 2}$ is bounded in probability. In addition, if $S_{n} /\left(2 s_{n, p}^{2} L_{2} s_{n, p}^{p}\right)^{1 / 2} \rightarrow 0$ in probability,

$$
\limsup _{n \rightarrow \infty} \frac{\left\|S_{n}\right\|}{\left(2 s_{n, p}^{2} L_{2} s_{n, p}^{p}\right)^{1 / 2}} \leq\left\{\begin{array}{ll}
2^{1+1 / p} & \text { for } 1 \leq p<2 \\
1 & \text { for } p=2
\end{array}\right. \text { a.s. }
$$


Now we discuss the result for i.i.d. $B$-valued random variables.

Theorem 2.3. Let $\left\{X_{n}, n \geq 1\right\}$ be a sequence of i.i.d. B-valued random variables with $X \in W M_{0}^{p}$ for some $p \in[1,2]$. Let $\left\{c_{n}, n \geq 1\right\}$ be a sequence of positive numbers satisfying

$$
c_{n} \geq n^{1 / p}\left(L_{2} n\right)^{1 / 2} .
$$

Then for some $\Gamma>0$,

$$
\limsup _{n \rightarrow \infty} \frac{\left\|S_{n}\right\|}{c_{n}} \leq \Gamma \quad \text { a.s. }
$$

holds provided that

$$
S_{n} / c_{n} \rightarrow 0 \quad \text { in probability }
$$

and

In particular,

$$
\sum_{n=1}^{\infty} P\left(\left\|X_{1}\right\| \geq c_{n}\right)<+\infty
$$

$$
\limsup _{n \rightarrow \infty} \frac{\left\|S_{n}\right\|}{\left(2 n^{2 / p} L_{2} n\right)^{1 / 2}} \leq\left\{\begin{array}{ll}
2^{1+1 / p} \sigma_{p} & \text { for } 1 \leq p<2 \\
\sigma_{2} & \text { for } p=2
\end{array}\right. \text { a.s. }
$$

holds provided that

$$
S_{n} /\left(2 n^{2 / p} L_{2} n\right)^{1 / 2} \rightarrow 0 \quad \text { in probability }
$$

and

$$
E \frac{\left\|X_{1}\right\|^{p}}{\left(L_{2}\left\|X_{1}\right\|\right)^{p / 2}}<+\infty
$$

where $\sigma_{p}=\sup _{f \in B_{1}^{*}}\left(E|f(X)|^{p}\right)^{1 / p}$.

Next we state the results for $r=\frac{1}{q}=\frac{p}{p-1}$.

Theorem 2.4. Let $\left\{X_{n}, n \geq 1\right\}$ be a sequence of independent B-valued random variables with $X_{n} \in W M_{0}^{p}$ for some $p \in[1,2]$. Assuming the following statements hold:

$$
\begin{gathered}
\lim _{n \rightarrow \infty} s_{n, p}^{p}=+\infty \\
\left\|X_{n}\right\| \leq \eta_{n} s_{n, p} /\left(L_{2} s_{n, p}^{p}\right)^{1 / p} \quad \text { a.s. }
\end{gathered}
$$

for some sequence of real number $\left\{\eta_{n}\right\}$ with $1 \geq \eta_{n} \rightarrow 0$, and

$$
S_{n} /\left(2 s_{n, p}^{q} L_{2} s_{n, p}^{p}\right)^{1 / q} \rightarrow 0 \text { in probability. }
$$

Then

$$
\limsup _{n \rightarrow \infty} \frac{\left\|S_{n}\right\|}{\left(2 s_{n, p}^{q} L_{2} s_{n, p}^{p}\right)^{1 / q}} \leq \Lambda_{p} \text { a.s. }
$$

where

$$
\Lambda_{p}= \begin{cases}0 & \text { for } p=1 \\ \left(16 K_{0}\right)^{1 / p} q^{1 / q} & \text { for } 1<p<2 \\ 1 & \text { for } p=2\end{cases}
$$

and $K_{0}$ is a universal constant in isoperimetric inequality in Talagrand [12]. 
Theorem 2.5. Let $\left\{X_{n}, n \geq 1\right\}$ be a sequence of independent B-valued random variables with $X_{n} \in W M_{0}^{p}$ for some $p \in[1,2]$. Suppose that the following statements hold:

$$
\begin{gathered}
\sum_{n=1}^{\infty}\left(s_{n, p}^{q} L_{2} s_{n, p}^{p}\right)^{-\alpha / q} E\left\|X_{n}\right\|^{\alpha}<+\infty \quad \text { for some } \alpha>p ; \\
s_{n, p} \rightarrow \infty
\end{gathered}
$$

Then

$$
\limsup _{n \rightarrow \infty} \frac{\left\|S_{n}\right\|}{\left(2 s_{n, p}^{q} L_{2} s_{n, p}^{p}\right)^{1 / q}} \leq \Gamma \quad \text { a.s. for some } \Gamma>0
$$

holds provided that $S_{n} /\left(2 s_{n, p}^{q} L_{2} s_{n, p}^{p}\right)^{1 / q}$ is bounded in probability. In addition, if $S_{n} /\left(2 s_{n, p}^{q} L_{2} s_{n, p}^{p}\right)^{1 / q} \rightarrow 0$ in probability,

$$
\limsup _{n \rightarrow \infty} \frac{\left\|S_{n}\right\|}{\left(2 s_{n, p}^{q} L_{2} s_{n, p}^{p}\right)^{1 / q}} \leq \Lambda_{p} \text { a.s. }
$$

where $\Lambda_{p}$ is the same as that in Theorem 2.4.

Similar to Theorem 2.3, we have the following result.

Theorem 2.6. Let $\left\{X_{n}, n \geq 1\right\}$ be a sequence of i.i.d. B-valued random variables with $X \in W M_{0}^{p}$ for some $p \in[1,2]$. Let $\left\{c_{n}, n \geq 1\right\}$ be a sequence of positive numbers satisfying

$$
c_{n} \geq n^{1 / p}\left(L_{2} n\right)^{1 / q} .
$$

Then for some $\Gamma>0$,

$$
\limsup _{n \rightarrow \infty} \frac{\left\|S_{n}\right\|}{c_{n}} \leq \Gamma \quad \text { a.s. }
$$

holds provided that (2.7) and (2.8) hold. In particular,

$$
\limsup _{n \rightarrow \infty} \frac{\left\|S_{n}\right\|}{\left(2 n^{q / p} L_{2} n\right)^{1 / q}} \leq \Lambda_{p} \sigma_{p} \text { a.s. }
$$

holds provided that

$$
S_{n} /\left(2 n^{q / p} L_{2} n\right)^{1 / q} \rightarrow 0 \quad \text { in probability }
$$

and

$$
E \frac{\left\|X_{1}\right\|^{p}}{\left(L_{2}\left\|X_{1}\right\|\right)^{p-1}}<+\infty
$$

where $\sigma_{p}=\sup _{f \in B_{1}^{*}}\left(E|f(X)|^{p}\right)^{1 / p}$ and $\Lambda_{p}$ is the same as that in Theorem 2.4.

Remarks. (1) The above theorems answer the questions proposed in Section 1 for normalized constants $s_{n, p}\left(L_{2} s_{n, p}^{p}\right)^{1 / 2}$ and $s_{n, p}\left(L_{2} s_{n, p}^{p}\right)^{\frac{p-1}{p}}$ and, give an accurate estimate of the upper limit for the nonrandom constant. In particular, for $p=2$, Theorems 2.1 and 2.4 are versions of Kolmogorov's BLIL, Theorems 2.2 and 2.5 are the versions of Wittmann's BLIL for $B$-valued random variables and Theorems 2.3 and 2.6 is 
the versions of BLIL for i.i.d. $B$-valued random variables. In that sense, our results are the extensions of the BLIL for $B$-valued random variables. Also, some results are new even for real valued random variables. Since $s_{n, p} \leq\left(\sum_{i=1}^{n} E\left\|X_{i}\right\|^{p}\right)^{1 / p}$, the similar results may be obtained for the normalizer constant $\left(\sum_{i=1}^{n} E\left\|X_{i}\right\|^{p}\right)^{1 / p}\left(L_{2} \sum_{i=1}^{n} E\left\|X_{i}\right\|^{p}\right)^{1 / 2}$.

(2) In Theorems 2.2 and 2.5, the assumptions that Conditions (2.4) and (2.14) holds "for some $\alpha>p$ " can not be strengthened to say "for some $\alpha \geq p$ ". In fact (2.4) and (2.14) fail for $s_{n, p}$ when $\alpha=p$. Indeed, if (2.4) holds with $\alpha=p$, that is,

$$
\sum_{n=1}^{\infty}\left(s_{n, p}^{2} L_{2} s_{n, p}^{p}\right)^{-p / 2} E\left\|X_{n}\right\|^{p}<+\infty,
$$

then defining $n_{k}$ to be the smallest positive integer satisfying that $s_{n, p}^{p}>\rho^{k}, k \geq 1$, where $\rho>1$, we have that

$$
\left(s_{n_{k+1}, p}^{2} L_{2} s_{n_{k+1}, p}^{p}\right)^{p / 2}\left(s_{n_{k}, p}^{2} L_{2} s_{n_{k}, p}^{p}\right)^{-p / 2} \sim \rho, \quad \text { and } \quad s_{n_{k}, p}^{p} \sim \rho^{k}
$$

Hence, setting $\gamma_{i}=\sup \left\{k: i \geq n_{k}\right\}$,

$$
\begin{aligned}
\sum_{k=1}^{\infty} \frac{1}{\left(s_{n_{k}, p}^{2} L_{2} s_{n_{k}, p}^{p}\right)^{p / 2}} \sum_{i=1}^{n_{k}} E\left\|X_{i}\right\|^{p} & \leq \sum_{j=1}^{\infty} E\left\|X_{i}\right\|^{p} \sum_{k=\gamma_{j}}^{\infty} \frac{1}{\left(s_{n_{k}, p}^{2} L_{2} s_{n_{k}, p}^{p}\right)^{p / 2}} \\
& \leq \sum_{j=1}^{\infty} E\left\|X_{j}\right\|^{p} \sum_{k=\gamma_{j}}^{\infty} \frac{1}{\left(s_{n_{\gamma_{i}}, p}^{2} L_{2} s_{n_{\gamma_{i}}, p}^{p}\right)^{p / 2} \rho^{\left(k-\gamma_{j}\right)}} \\
& \leq \sum_{j=1}^{\infty} \frac{E\left\|X_{j}\right\|^{r}}{\left(s_{j, p}^{2} L_{2} s_{j, p}^{p}\right)^{p / 2}} \sum_{k=0}^{\infty} \frac{1}{\rho^{k}} \\
& \leq\left(1-\frac{1}{\rho}\right)^{-1} \sum_{j=1}^{\infty} \frac{E\left\|_{j}\right\|^{r}}{\left(s_{j, p}^{2} L_{2} s_{j, p}^{p}\right)^{p / 2}}<+\infty .
\end{aligned}
$$

On the other hand,

$$
\begin{aligned}
& \sum_{k=1}^{\infty} \frac{1}{\left(s_{n_{k}, p}^{2} L_{2} s_{n_{k}, p}^{p}\right)^{p / 2}} \sum_{i=1}^{n_{k}} E\left\|X_{i}\right\|^{p} \geq \\
& \sum_{k=1}^{\infty} \frac{1}{s_{n_{k}, p}^{p}\left(L_{2} s_{n_{k}, p}^{p}\right)^{p / 2}} \sum_{i=1}^{n_{k}} E\left\|X_{i}\right\|^{p} \geq \sum_{k=1}^{\infty}\left(L_{2} s_{n_{k}, p}^{p}\right)^{-1} \geq \sum_{k=1}^{\infty}(\log (k \log \rho))^{-1}=+\infty
\end{aligned}
$$

which results in a contradiction.

(3) Obviously, for $1<p<2$, the upper limits in the above theorems are not best and perhaps can be improved. On the other hand, the lower limits for the nonrandom constants do not be given and it is unknown whether the lower limits are greater than zero or not.

(4) Furthermore, from Theorem 2.6, the law of large number is obtained for $p=1$ and the LIL is obtained for $p=2$. The conjecture is that there exists an continuous increasing function $\Lambda(p)$ such that $\Lambda(0)=0, \Lambda(2)=1$ and for i.i.d. $B$-valued random variables such that

$$
\limsup _{n \rightarrow \infty}\left\|S_{n}\right\| /\left(2 n^{q / p} L_{2} n\right)^{1 / q}=\Lambda(p) \text { a.s. }
$$




\section{The Proofs of theorems}

Now we first prove Theorem 2.1.

\section{The proof of Theorem 2.1.}

By the Hoffmann-Jørgensen inequality and the standard method of symmetrization, it is easy to prove that (2.2) and (2.3) imply that

$$
\lim _{n \rightarrow \infty} \frac{E\left\|S_{n}\right\|}{s_{n, p} t_{n}}=\lim _{n \rightarrow \infty} \frac{E\left\|\sum_{i=1}^{n} \epsilon_{i} X_{i}\right\|}{s_{n, p} t_{n}}=0,
$$

where $t_{n}=\left(2 L_{2} s_{n, p}^{p}\right)^{1 / 2}$ and $\left\{\epsilon_{n}, n \geq 1\right\}$ is a Rademacher sequence which is independent of $\left\{X_{n}, n \geq 1\right\}$.

For $\rho>1$, let $n_{k}=\min \left\{n ; s_{n, p}>\rho^{k}\right\}$. Then, $s_{n_{k}, p} \sim \rho^{k}$, and $s_{n_{k+1}, p} / s_{n_{k}, p} \sim \rho$. To the claim of Theorem 2.1, it will be sufficient to show that for every $\epsilon>0$ and some $\rho>1$,

$$
\sum_{k=1}^{\infty} P\left\{\left\|S_{n_{k}}\right\|>\left(2^{1+1 / p}+\epsilon\right) s_{n_{k}, p} t_{n_{k}}\right\}<+\infty .
$$

Let now $\epsilon>0$ and $\rho>1$ be fixed. For $f, g \in D$ and every $k$, set

$$
d_{2}^{k}(f, g)=\frac{1}{s_{n_{k}, p}}\left(\sum_{i=1}^{n_{k}} E(f-g)^{2}\left(X_{i}\right)\right)^{1 / 2}
$$

and

$$
\beta_{k}=E\left\|\sum_{i=1}^{n_{k}} \epsilon_{i} X_{i}\right\| /\left(s_{n_{k}, p} t_{n_{k}}\right) .
$$

By $(3.1), \beta_{k} \rightarrow 0$ as $k \rightarrow \infty$.

Let $N\left(D, d_{2}^{k}, \epsilon\right)$ denote the entropy, that is, $N\left(D, d_{2}^{k}, \epsilon\right)$ is the minimal number of elements $g$ in $D$ such that for every $f \in D$ there exists such a $g$ with $d_{2}^{k}(f, g)<\epsilon$. By Theorem 2 in Chow and Teicher [5] and von Bahr-Esseen's inequality (see von Bahr and Esseen [2], Th. 4), we have that for every $f \in B_{1}^{*}$,

$$
\begin{aligned}
& {\left[\sum_{i=1}^{n} E f^{2}\left(X_{i}\right)\right]^{1 / 2}=\left[E\left|\sum_{i=1}^{n} f\left(X_{i}\right)\right|^{2}\right]^{1 / 2} \leq A_{1}^{-1} E\left|\sum_{i=1}^{n} f\left(X_{i}\right)\right|} \\
& \leq A_{1}^{-1}\left(2^{p-1} \sum_{i=1}^{n} E\left|f\left(X_{i}\right)\right|^{p}\right)^{1 / p} \leq 2^{1+1 / p}\left(\sum_{i=1}^{n} E\left|f\left(X_{i}\right)\right|^{p}\right)^{1 / p}
\end{aligned}
$$

and thus,

$$
\sigma_{n}=\left(\sup _{f \in B_{1}^{*}} \sum_{i=1}^{n} E f^{2}\left(X_{i}\right)\right)^{1 / 2} \leq 2^{1+1 / p} s_{n, p} .
$$

By using Proposition 4.13 in Ledoux and Talagrand [14], the argument like the proof of Lemma 8.3 in Ledoux and Talagrand [14] and (3.3), one may prove that

$$
N\left(D, d_{2}^{k}, \epsilon\right) \leq \exp \left(\beta_{k} t_{n_{k}}^{2}\right)
$$

Now we establish (3.2). According to (3.4), we denote, for each $k$ (large enough) and $f$ in $D$, by $g_{k}(f)$ an element of $D$ such that $d_{2}^{k}(f, g)<\epsilon$ in such a way that set $D_{k}$ of all $g_{k}(f)$ 's has a cardinality less $\operatorname{than} \exp \left(\beta_{k} t_{n_{k}}^{2}\right)$. 
Then we have that

$$
\left\|\sum_{i=1}^{n_{k}} X_{i}\right\| \leq \sup _{g \in D_{k}}\left|\sum_{i=1}^{n_{k}} g\left(X_{i}\right)\right|+\sup _{h \in D_{k}^{\prime}}\left|\sum_{i=1}^{n_{k}} h\left(X_{i}\right)\right|
$$

where $D_{k}^{\prime}=\left\{f-g_{k}(f): f \in D\right\}$. The main observation concerning $D_{k}^{\prime}$ is that

$$
\sup _{h \in D_{k}^{\prime}}\left[\sum_{i=1}^{n_{k}} E\left|h\left(X_{i}\right)\right|^{2}\right)^{1 / 2} \leq \epsilon s_{n_{k}, p} .
$$

In order that (3.2) holds, it is sufficient to show that for some constant

$$
\sum_{k} P\left\{\sup _{h \in D_{k}^{\prime}}\left|\sum_{i=1}^{n_{k}} h\left(X_{i}\right)\right|>M \epsilon s_{n_{k}, p} t_{n_{k}}\right\}<\infty
$$

and

$$
\sum_{k} P\left\{\sup _{g \in D_{k}}\left|\sum_{i=1}^{n_{k}} g\left(X_{i}\right)\right|>\left(2^{1+1 / p}+\epsilon\right) s_{n_{k}, p} t_{n_{k}}\right\}<\infty .
$$

To prove (3.5), replacing $\left\{X_{i}\right\}$ by $\left\{X_{i}-X_{i}^{\prime}\right\}$ where $\left\{X_{i}^{\prime}\right\}$ is an independent copy of the sequence $\left\{X_{i}\right\}$ and noting that

$$
s_{n, p} \leq \sup _{f \in D}\left(\sum_{i=1}^{n} E\left|f\left(X_{i}-X_{i}^{\prime}\right)\right|^{p}\right)^{1 / p} \leq 2^{\frac{p-1}{p}} s_{n, p},
$$

we can assume the symmetrization of random variables. By taking $q=2 K_{0}, k=\left[t_{n_{k}}^{2}\right]+1, s=2 \epsilon s_{n_{k}, p} t_{n_{k}}, t=$ $M^{\prime} \epsilon s_{n_{k}, p} t_{n_{k}}$ and using proposition 1.1 in Ledoux and Talagrand [13], we have that

$$
\begin{aligned}
P\left\{\sup _{h \in D_{k}^{\prime}}\left|\sum_{i=1}^{n_{k}} h\left(X_{i}\right)\right|>M \epsilon s_{n_{k}, p} t_{n_{k}}\right\} \leq & \left(\frac{1}{2}\right)^{t_{n_{k}}^{2}}+P\left\{\sum_{i=1}^{\left[t_{n_{k}}^{2}\right]+1}\left\|X_{i}\right\|^{*}>2 \epsilon s_{n_{k}, p} t_{n_{k}}\right\} \\
& +4 \exp \left\{-\frac{\left(M^{\prime} \epsilon s_{n_{k}, p} t_{n_{k}}\right)^{2}}{128 K_{0} \sigma_{k}^{2}}\right\}+4 \exp \left\{-\frac{\left(M^{\prime} \epsilon s_{n_{k}, p} t_{n_{k}}\right)^{2} t_{n_{k}}^{2}}{768 M_{k} 2 s_{n_{k}, p} t_{n_{k}}}\right\}
\end{aligned}
$$

where

$$
\sigma_{k}=\sup _{h \in D_{k}^{\prime}}\left(\sum_{i=1}^{n_{k}} E h^{2}\left(X_{i} I\left\{\left\|X_{i}\right\| \leq s / k\right\}\right)\right)^{1 / 2} \leq \sup _{h \in D_{k}^{\prime}}\left(\sum_{i=1}^{n_{k}} E h^{2}\left(X_{i}\right)\right)^{1 / 2} \leq \epsilon s_{n_{k}, p}
$$

and

$$
\frac{M_{k}}{s_{n_{k}, p} t_{n_{k}}}=\frac{E\left\|\sum_{i=1}^{n_{k}} X_{i} I\left\{\left\|X_{i}\right\| \leq s / k\right\}\right\|}{s_{n_{k}, p} t_{n_{k}}} \leq \frac{E\left\|\sum_{i=1}^{n_{k}}\right\|}{s_{n_{k}, p} t_{n_{k}}} \rightarrow 0 .
$$

Noting that for large $k$,

$$
\sum_{i=1}^{\left[t_{n_{k}}^{2}\right]+1}\left\|X_{i}\right\|^{*} \leq\left(\left[t_{n_{k}}^{2}\right]+1\right) \max _{i \leq n_{k}}\left\|X_{i}\right\| \leq\left(\left[t_{n_{k}}^{2}\right]+1\right) \epsilon s_{n_{k}, p} / t_{n_{k}} \leq 2 \epsilon s_{n_{k}, p} t_{n_{k}}
$$


we have that

$$
P\left\{\sum_{i=1}^{\left[t_{n_{k}}^{2}\right]+1}\left\|X_{i}\right\|^{*}>2 \epsilon s_{n_{k}, p} t_{n_{k}}\right\}=0 .
$$

Thus, we have that for $M\left(M^{\prime}\right)$ large enough,

$$
\begin{aligned}
P\left\{\sup _{h \in D_{k}^{\prime}}\left|\sum_{i=1}^{n_{k}} h\left(X_{i}\right)\right|>M \epsilon s_{n_{k}, p} t_{n_{k}}\right\} & \leq 2^{-t_{n_{k}}^{2}}+4 \exp \left\{-\frac{M^{\prime 2} \epsilon^{2} s_{n_{k}, p}^{2}}{128 K_{0} \epsilon^{2} s_{n_{k}, p}^{2}} t_{n_{k}}^{2}\right\}+4 \exp \left\{-\frac{M^{\prime 2} \epsilon^{2}}{1536 \frac{M_{k}}{s_{n_{k}, p} t_{n_{k}}}} t_{n_{k}}^{2}\right\} \\
& \leq 2^{-t_{n_{k}}^{2}}+4 \exp \left\{-\frac{M^{\prime 2}}{128 K_{0}} t_{n_{k}}^{2}\right\}+4 \exp \left\{-\frac{M^{\prime 2}}{1536} t_{n_{k}}^{2}\right\} \\
& \leq 2^{-2 L_{2} \rho^{k p}}+8 \exp \left\{-2 \log _{2} \rho^{k p}\right\} .
\end{aligned}
$$

This completes the proof of (3.5). In order to prove (3.6), by using Lemma 1.6 in Ledoux and Talagrand [14] and noting $\sigma_{n}^{2} \leq 2^{2+2 / p} s_{n, p}^{2}$, for all large enough $k$ 's

$$
\begin{aligned}
& P\left\{\left|\sum_{i=1}^{n_{k}} g\left(X_{i}\right)\right|>\left(2^{1+1 / p}+\epsilon\right) s_{n_{k}, p} t_{n_{k}}\right\} \\
& \leq 2 \exp \left\{-\frac{\left(2^{1+1 / p}+\epsilon\right)^{2} s_{n_{k}, p}^{2} t_{n_{k}}^{2}}{2 \sigma_{n_{k}}^{2}}\left(2-\exp \left[\frac{\left(2^{1+1 / p}+\epsilon\right) s_{n_{k}, p} t_{n_{k}} \frac{\eta_{n_{k}} s_{n_{k}, p}}{t_{n_{k}}}}{\sigma_{n_{k}}^{2}}\right]\right)\right\} \\
& \leq 2 \exp \left\{-\frac{\left(2^{1+1 / p}+\epsilon\right)^{2} s_{n_{k}, p}^{2} 2 L_{2} s_{n_{k}, p}^{p}}{2^{3+2 / p} s_{n_{k}, p}^{2}}\left(2-\exp \left[\frac{\left(2^{1+1 / p}+\epsilon\right) \eta_{n_{k}} s_{n_{k}, p}^{2}}{2^{2+2 / p} s_{n_{k}, p}^{2}}\right]\right)\right\} \\
& \leq 2 \exp \left\{-\left(1+\frac{\epsilon}{4}\right) \log _{2} s_{n_{k}, p}^{p}\right\} .
\end{aligned}
$$

Hence for $k_{0}$ large enough,

$$
\begin{aligned}
\sum_{k \geq k_{0}} P\left\{\sup _{g \in D_{k}}\left|\sum_{i=1}^{n_{k}} g\left(X_{i}\right)\right|>\left(2^{1+1 / p}+\epsilon\right) s_{n_{k}, p} t_{n_{k}}\right\} & \leq \sum_{k \geq k_{0}} 2 \operatorname{card}\left(D_{k}\right) \exp \left\{-\left(1+\frac{\epsilon}{4}\right) \log _{2} s_{n_{k}, p}^{p}\right\} \\
& \leq \sum_{k \geq k_{0}} 2 \exp \left\{2 \beta_{k} \log _{2} s_{n_{k}, p}^{p}\right\} \exp \left\{-\left(1+\frac{\epsilon}{4}\right) \log _{2} s_{n_{k}, p}^{p}\right\} \\
& \leq 2 \sum_{k \geq k_{0}} \exp \left\{-\left(1+\frac{\epsilon}{8}\right) \log _{2} s_{n_{k}, p}^{p}\right\}<+\infty
\end{aligned}
$$

The proof of Theorem 2.1 is complete.

Now we are in the position to prove Theorem 2.2. In order to establish this theorem, we need the following proposition.

Proposition 3.1. Let $\left\{X_{n}, n \geq 1\right\}$ be a sequence of independent B-valued random variables. Suppose that for a positive sequence of real numbers $\left\{a_{n}, n \geq 1\right\}$ with $a_{n} \uparrow+\infty$ and a real number $\alpha \geq 1$, the following statements hold

$$
\begin{aligned}
& \sum_{n=1}^{\infty} a_{n}^{-\alpha} E\left\|X_{n}\right\|^{\alpha}<\infty, \\
& \limsup _{n \rightarrow \infty} a_{n+1} / a_{n}<+\infty .
\end{aligned}
$$


(1) For $1 \leq \alpha \leq 2$ and $X_{n} \in W M_{0}^{\alpha}$,

$$
\limsup _{n \rightarrow \infty}\left\|S_{n}\right\| / a_{n}=\left\{\begin{array}{ll}
0 & \\
\Gamma & \text { for some } \Gamma>0,
\end{array}\right. \text { a.s. }
$$

if and only if

$$
\limsup _{n \rightarrow \infty}\left\|S_{n}\right\| / a_{n}=\left\{\begin{array}{l}
0 \\
M \quad \text { for some } M>0,
\end{array}\right. \text { in probability }
$$

respectively.

(2) Assume, moreover, that there is some $\beta>0$ such that for any $n \geq 1$

$$
1 \leq a_{n+1} / a_{n} \leq\left(s_{n+1, p} / s_{n, p}\right)^{\beta}
$$

and for some $1 \leq p \leq 2, X_{n} \in W M_{0}^{p}$ and

$$
\limsup _{n \rightarrow \infty}\left(s_{n, p}^{2} L_{2} s_{n, p}^{p}\right)^{1 / 2} / a_{n}<+\infty .
$$

Then for $\alpha>2$,

$$
\limsup _{n \rightarrow \infty}\left\|S_{n}\right\| / a_{n}<+\infty \text { a.s. }
$$

if and only if

$$
\limsup _{n \rightarrow \infty}\left\|S_{n}\right\| / a_{n}<+\infty \text { in probability. }
$$

In addition, assume that

$$
\limsup _{n \rightarrow \infty}\left(s_{n, p}^{2} L_{2} s_{n, p}^{p}\right)^{1 / 2} / a_{n}=0 .
$$

Then,

if and only if

$$
\limsup _{n \rightarrow \infty}\left\|S_{n}\right\| / a_{n}=0 \text { a.s. }
$$

$$
\limsup _{n \rightarrow \infty}\left\|S_{n}\right\| / a_{n}=0 \text { in probability. }
$$

Proof. We only prove that under the Conditions (3.7) and (3.8), (3.10) implies (3.9). As in the proof of Theorem 2.1, it can be shown that (3.10) implies that

$$
\limsup _{n \rightarrow \infty} E\left\|S_{n}\right\| / a_{n}\left\{\begin{array}{l}
=0 \\
<+\infty .
\end{array}\right.
$$

Set $\rho>D=\limsup _{n \rightarrow \infty} a_{n+1} / a_{n}$. For $k \geq 1$, let $n_{k}$ be the smallest positive integer satisfying that $a_{n}>\rho^{k}$. it is easy to see that

$$
a_{n_{k}} \sim \rho^{k} \quad \text { and } \quad a_{n_{k+1}} / a_{n_{k}} \sim \rho .
$$

By the standard argument, in order to prove that $\limsup _{n \rightarrow \infty}\left\|\sum_{i=1}^{n} X_{i}\right\| / a_{n}=0(=\Gamma)$ a.s., it is sufficient to prove that for $\forall \epsilon>0$ (or for some $\epsilon>0$ ),

$$
\sum_{k=1}^{\infty} P\left\{\left\|\sum_{i=n_{k}+1}^{n_{k+1}} X_{i}\right\| \geq \epsilon a_{n_{k+1}}\right\}<+\infty .
$$


By (3.18), for $k$ large enough, $E\left\|\sum_{i=n_{k}+1}^{n_{k+1}} X_{i}\right\| / a_{n_{k+1}} \leq \epsilon / 2$. Thus, by de Acosta's inequality (see de Acosta [1]), for sufficiently large $k$,

$$
\begin{aligned}
P\left\{\left\|\sum_{i=n_{k}+1}^{n_{k+1}} X_{i}\right\| \geq \epsilon a_{n_{k+1}}\right\} & \leq P\left\{\left\|\sum_{i=n_{k}+1}^{n_{k+1}} X_{i}\right\|-E\left\|\sum_{i=n_{k}+1}^{n_{k+1}} X_{i}\right\| \geq \frac{\epsilon}{2} a_{n_{k+1}}\right\} \\
& \leq \frac{2^{\alpha}}{\epsilon^{\alpha} a_{n_{k+1}}^{\alpha}} \sum_{i=n_{k}+1}^{n_{k+1}} E\left\|X_{i}\right\|^{\alpha} \leq \frac{2^{\alpha}}{\epsilon^{\alpha}} \sum_{i=n_{k}+1}^{n_{k+1}} \frac{E\left\|X_{i}\right\|^{\alpha}}{a_{i}^{\alpha}} .
\end{aligned}
$$

Therefore,

$$
\sum_{k=1}^{\infty} P\left\{\left\|\sum_{i=1}^{n_{k}} X_{i}\right\| \geq \epsilon a_{n_{k+1}}\right\}<+\infty
$$

The proof of Part (1) is complete.

Now we are in the position to prove Part (2). We only prove that under the Conditions (3.7), (3.11) and (3.15), (3.17) implies (3.16). Now, by Lemma 3.3 in Wittmann [15], for any $M>1$, there exists a strictly increasing subsequence of positive integers such

$$
M a_{n_{k}} \leq a_{n_{k+1}} \leq M^{3} a_{n_{k}+1}
$$

From Proposition 2 of Chen [3], it is sufficient to show that

$$
\sum_{k=1}^{\infty} \exp \left\{-\frac{\delta a_{n_{k}}^{2}}{\sigma_{k}^{2}}\right\}<+\infty \quad \text { for every } \delta>0
$$

where $\sigma_{k}^{2}=\sup _{f \in S_{1}^{*}} \sum_{i=1}^{n_{k}} E f^{2}\left(X_{i}\right)(k \geq 1)$. Now from (3.11), $s_{n_{k+1}, p} / s_{n_{k}, p} \sim M^{1 / \beta}$ and from which, (3.15) and (3.3), it is easy to see that for sufficiently large $k$,

$$
\frac{\delta a_{n_{k}}}{\sigma_{k}^{2}} \geq \frac{\delta^{\prime} s_{n_{k}, p}^{2} L_{2} s_{n_{k}, p}^{p}}{\sigma_{k}^{2}} \geq \delta^{\prime \prime} L_{2} s_{n_{k}, p}^{p} \geq 2 \log \left(k \log M^{1 / \beta}\right)
$$

Hence (3.9) holds. This completes the proof of Proposition 3.1.

\section{The proof of Theorem 2.2}

The theorem follows from Proposition 3.1 for $1 \leq p<\alpha \leq 2$ and from Wittmann's LIL (see Chen [3]) for $\alpha>p=2$, respectively. We only prove that for $p<2<\alpha$, under the Conditions (2.4) and (2.5), $S_{n} /\left(2 s_{n, p}^{2} L_{2} s_{n, p}^{p}\right)^{1 / 2} \rightarrow 0$ in probability implies that

$$
\limsup _{n \rightarrow \infty} \frac{\left\|S_{n}\right\|}{\left(2 s_{n, p}^{2} L_{2} s_{n, p}^{p}\right)^{1 / 2}} \leq 2^{1+1 / p} \text { a.s. }
$$

For this purpose, define

$$
\begin{aligned}
& X_{n}^{\prime}=X_{n} I\left\{\left\|X_{n}\right\|<\left(2 s_{n, p}^{2} L_{2} s_{n, p}^{p}\right)^{1 / 2}\right\}, \\
& X_{n}^{\prime \prime}=X_{n} I\left\{\left\|X_{n}\right\| \geq\left(2 s_{n, p}^{2} L_{2} s_{n, p}^{p}\right)^{1 / 2}\right\}=X_{n}-X_{n}^{\prime} .
\end{aligned}
$$


By (2.4),

$$
\sum_{n=1}^{\infty} P\left(\left\|X_{n}\right\| \geq\left(2 s_{n, p}^{2} L_{2} s_{n, p}^{p}\right)^{1 / 2}\right) \leq \sum_{n=1}^{\infty}\left(2 s_{n, p}^{2} L_{2} s_{n, p}^{p}\right)^{-\alpha / 2} E\left\|X_{n}\right\|^{\alpha}<+\infty .
$$

By the Borel-Cantelli Lemma,

$$
\lim _{n \rightarrow \infty}\left(2 s_{n, p}^{2} L_{2} s_{n, p}^{p}\right)^{-1 / 2} \sum_{i=1}^{n} X_{i}^{\prime \prime}=0 \quad \text { a.s. }
$$

and for $n \geq 1$,

$$
\begin{aligned}
& \left\|\sum_{i=1}^{n} E X_{i}^{\prime \prime}\right\|=\sup _{f \in B_{1}^{*}} \sum_{i=1}^{n} E f\left(X_{i}^{\prime \prime}\right) \\
& \leq \sup _{f \in B_{1}^{*}}\left(\sum_{i=1}^{n} E\left|f\left(X_{i}\right)\right|^{p}\right)^{1 / p}\left(\sum_{i=1}^{n} P\left(\left\|X_{i}\right\| \geq\left(2 s_{i, p}^{2} L_{2} s_{i, p}^{p}\right)^{1 / 2}\right)\right)^{\frac{p-1}{p}} \leq K s_{n, p} \rightarrow \infty \quad(n \rightarrow \infty)
\end{aligned}
$$

where $K=\left(\sum_{i=1}^{\infty} P\left(\left\|X_{i}\right\| \geq\left(2 s_{i, p}^{2} L_{2} s_{i, p}^{p}\right)^{1 / 2}\right)\right)^{\frac{p-1}{p}}$. Thus,

$$
\frac{\left\|\sum_{i=1}^{n} E X_{i}^{\prime \prime}\right\|}{\left(2 s_{n, p}^{2} L_{2} s_{n, p}^{p}\right)^{1 / 2}} \rightarrow 0 \quad(n \rightarrow \infty) .
$$

Since $\sum_{i=1}^{n} X_{i} /\left(2 s_{n, p}^{2} L_{2} s_{n, p}^{p}\right)^{1 / 2} \rightarrow 0$ in probability, by (3.22) and (3.23), we may obtain that

$$
\sum_{i=1}^{n}\left(X_{i}^{\prime}-E X_{i}^{\prime}\right) /\left(2 s_{n, p}^{2} L_{2} s_{n, p}^{p}\right)^{1 / 2} \rightarrow 0 \quad \text { in probability. }
$$

Again by (3.22) and (3.23), in order to prove the theorem, it is sufficient to show that

$$
\limsup _{n \rightarrow \infty} \frac{\left\|\sum_{i=1}^{n}\left(X_{i}^{\prime}-E X_{i}^{\prime}\right)\right\|}{\left(2 s_{n, p}^{2} L_{2} s_{n, p}^{p}\right)^{1 / 2}} \leq \Lambda_{1} \quad \text { a.s. }
$$

By (2.4) and Kronecker Lemma, we have that

$$
\left(2 s_{n, p}^{2} L_{2} s_{n, p}^{p}\right)^{-\alpha / 2} \sum_{i=1}^{n} E\left\|X_{i}\right\|^{\alpha} \rightarrow 0 \quad(n \rightarrow \infty) .
$$

Hence by the boundedness of $\left\{X_{i}^{\prime}-E X_{i}^{\prime}\right\}$ and (3.24), applying the standard method of symmetrization and Lemma 7.2 of Ledoux and Talagrand [14], we may obtain that

$$
\lim _{n \rightarrow \infty} \frac{E\left\|\sum_{i=1}^{n}\left(X_{i}^{\prime}-E X_{i}^{\prime}\right)\right\|}{\left(2 s_{n, p}^{2} L_{2} s_{n, p}^{p}\right)^{1 / 2}}=0 .
$$

By Lemma 3.3 of Wittmann [15], for any $M>1$, there exists a subsequence of positive integers $\left\{n_{k}\right\}$ with $M s_{n_{k}, p} \leq s_{n_{k+1}, p} \leq M^{3} s_{n_{k}+1, p}$. Thus, by the standard argument, to prove (3.25), it is enough to show that for $M>1$,

$$
\sum_{k=1}^{\infty} P\left\{\left\|\sum_{i=1}^{n_{k}}\left(X_{i}^{\prime}-E X_{i}^{\prime}\right)\right\|>\left(2^{1+1 / p}+4 \epsilon\right)\left(2 s_{n_{k}, p}^{2} L_{2} s_{n_{k}, p}^{p}\right)^{1 / 2}\right\}<+\infty .
$$


Now for $\alpha$ satisfying (2.4) and $\gamma_{j}=\inf \left\{k: j \leq n_{k}\right\}$, we have that

$$
\begin{aligned}
& \sum_{k=1}^{\infty} \frac{1}{\left(2 s_{n_{k}, p}^{2} L_{2} s_{n_{k}, p}^{p}\right)^{\alpha / 2}} \sum_{j=1}^{n_{k}} E\left\|X_{j}\right\|^{\alpha} \leq \sum_{j=1}^{\infty} E\left\|X_{j}\right\|^{\alpha} \sum_{k=\gamma_{j}}^{\infty} \frac{1}{\left(2 s_{n_{k}, p}^{2} L_{2} s_{n_{k}, p}^{p}\right)^{\alpha / 2}} \\
& \leq \sum_{j=1}^{\infty} E\left\|X_{j}\right\|^{\alpha} \sum_{k=\gamma_{j}}^{\infty} \frac{C}{\left(2 s_{n_{\gamma_{j}}, p}^{2} L_{2} s_{n_{\gamma_{j}}, p}\right)^{\alpha / 2} M^{\left(k-\gamma_{j}\right) r}} \\
& \leq \sum_{j=1}^{\infty} \frac{E\left\|X_{j}\right\|^{\alpha}}{\left(2 s_{j, p}^{2} L_{2} s_{j, p}^{p}\right)^{\alpha / 2}} \sum_{k=0}^{\infty} \frac{C}{M^{k r}} \\
& \leq C\left(1-\frac{1}{M^{r}}\right)^{-1} \sum_{j=1}^{\infty} \frac{E\left\|X_{j}\right\|^{\alpha}}{\left(2 s_{j, p}^{p} L_{2} s_{j, p}^{p}\right)^{\alpha / 2}} .
\end{aligned}
$$

It is obvious that (2.4) implies the finiteness of the right side. Thus we have proved that

$$
\sum_{k=1}^{\infty} \frac{1}{\left(2 s_{n_{k}, p}^{2} L_{2} s_{n_{k}, p}^{p}\right)^{\alpha / 2}} \sum_{j=1}^{n_{k}} E\left\|X_{j}\right\|^{\alpha}<+\infty
$$

By (3.29), one can find a sequence $\left\{\eta_{k}\right\}$ of positive numbers tending to zero such that

$$
\sum_{k=1}^{\infty}\left(\eta_{k}\left(2 s_{n_{k}, p}^{2} L_{2} s_{n_{k}, p}^{p}\right)^{1 / 2}\right)^{-\alpha} \sum_{j=1}^{n_{k}} E\left\|X_{j}\right\|^{\alpha}<+\infty .
$$

For $i \leq n_{k}$, define

$$
\begin{aligned}
& Y_{i k}=X_{i}^{\prime} I\left\{\left\|X_{i}^{\prime}\right\|<\frac{\eta_{k} s_{n_{k}, p}}{\left(2 L_{2} s_{n_{k}, p}^{p}\right)^{1 / 2}}\right\}=X_{i} I\left\{\left\|X_{i}\right\|<\frac{\eta_{k} s_{n_{k}, p}}{\left(2 L_{2} s_{n_{k}, p}^{p}\right)^{1 / 2}}\right\} \\
& Z_{i k}=X_{i}^{\prime} I\left\{\left\|X_{i}^{\prime}\right\| \geq \frac{\eta_{k} s_{n_{k}, p}}{\left(2 L_{2} s_{n_{k}, p}^{p}\right)^{1 / 2}}\right\}=X_{i} I\left\{\frac{\eta_{k} s_{n_{k}, p}}{\left(2 L_{2} s_{n_{k}, p}^{p}\right)^{1 / 2}} \leq\left\|X_{i}\right\|<\left(2 s_{i, p}^{2} L_{2} s_{i, p}^{p}\right)^{1 / 2}\right\} .
\end{aligned}
$$

Thus to prove (3.28), it sufficient to show that

$$
\sum_{k=1}^{\infty} P\left\{\left\|\sum_{i=1}^{n_{k}}\left(Y_{i k}-E Y_{i k}\right)\right\| \geq\left(2^{1+1 / p}+3 \epsilon\right)\left(2 s_{n_{k}, p}^{2} L_{2} s_{n_{k}, p}^{p}\right)^{1 / 2}\right\}<+\infty
$$

and

$$
\sum_{k=1}^{\infty} P\left\{\left\|\sum_{i=1}^{n_{k}}\left(Z_{i k}-E Z_{i k}\right)\right\| \geq \epsilon\left(2 s_{n_{k}, p}^{2} L_{2} s_{n_{k}, p}^{p}\right)^{1 / 2}\right\}<+\infty .
$$

Now we first prove (3.32). By Lemma 2.5 of Gine and Zinn [8], it is enough to show that for each $\epsilon>0$,

$$
\sum_{k=1}^{\infty} P\left\{\left\|\sum_{i=1}^{n_{k}} \epsilon_{i} Z_{i k}\right\|>\epsilon\left(2 s_{n_{k}, p}^{2} L_{2} s_{n_{k}, p}^{p}\right)^{1 / 2}\right\}<+\infty
$$

This is equivalent to showing, by the Hoffmann-Jørgensen's inequality (Ledoux and Talagrand [12] Prop. 2.1), that for $\forall \epsilon>0$,

$$
\sum_{k=1}^{\infty} P\left\{\max _{i \leq n_{k}}\left\|Z_{i k}\right\|>\epsilon\left(2 s_{n_{k}, p}^{2} L_{2} s_{n_{k}, p}^{p}\right)^{1 / 2}\right\}<+\infty
$$


and

$$
\sum_{k=1}^{\infty}\left(P\left\{\left\|\sum_{i=1}^{n_{k}} \epsilon_{i} Z_{i k}\right\|>\epsilon\left(2 s_{n_{k}, p}^{2} L_{2} s_{n_{k}, p}^{p}\right)^{1 / 2}\right\}\right)^{2}<+\infty .
$$

It is easy to see that (3.29) implies (3.34). Thus, to prove (3.35), by taking $q=\left[2 K_{0}\right]+1, m_{k}=\left[\delta_{k}^{-\frac{1}{2}}\right]$ (where $\delta_{k}=\left(\eta_{k}\right)^{-\alpha}\left(2 s_{n_{k}, p}^{2} L_{2} s_{n_{k}, p}^{p}\right)^{-\alpha / 2} \sum_{i=1}^{n_{k}} E\left\|X_{i}\right\|^{\alpha}, s=\eta_{k}\left(2 s_{n_{k}, p}^{2} L_{2} s_{n_{k}, p}^{p}\right)^{1 / 2}$ and $t=\frac{\epsilon}{3}\left(2 s_{n_{k}, p}^{2} L_{2} s_{n_{k}, p}^{p}\right)^{1 / 2}$ and applying Proposition 1.1 in Ledoux and Talagrand [13], we have that for some $\delta>0$,

$$
\begin{aligned}
& P\left\{\left\|\sum_{i=1}^{n_{k}} \epsilon_{i} Z_{i k}\right\|>\left(\frac{\epsilon}{3}+2 \eta_{k}\right)\left(2 s_{n_{k}, p}^{2} L_{2} s_{n_{k}, p}^{p}\right)^{1 / 2}+8 q M_{k}\right\} \\
& \leq\left(\frac{1}{2}\right)^{m_{k}}+P\left\{\sum_{i=1}^{m_{k}}\left\|Z_{i k}\right\|^{*}>\eta_{k}\left(2 s_{n_{k}, p}^{2} L_{2} s_{n_{k}, p}^{p}\right)^{1 / 2}\right\} \\
& +4 \exp \left\{-\frac{\left(\frac{\epsilon}{3}\right)^{2} 2 s_{n_{k}, p}^{2} L_{2} s_{n_{k}, p}^{p}}{64\left(\left[2 K_{0}\right]+1\right) \sigma_{k}^{2}}\right\}+4 \exp \left\{-\frac{\left(\frac{\epsilon}{3}\right)^{2} 2 m_{k} s_{n_{k}, p}^{2} L_{2} s_{n_{k}, p}^{p}}{768 M_{k} \eta_{k}\left(2 s_{n_{k}, p}^{2} L_{2} s_{n_{k}, p}^{p}\right)^{1 / 2}}\right\} \\
& \leq\left(\frac{1}{2}\right)^{m_{k}}+\frac{m_{k} E \max _{i \leq n_{k}}\left\|Z_{i k}\right\|^{\alpha}}{\eta_{k}^{\alpha}\left(2 s_{n_{k}, p}^{2} L_{2} s_{n_{k}, p}^{p}\right)^{\alpha / 2}}+4 \exp \left\{-\frac{\delta^{2}\left(2 s_{n_{k}, p}^{2} L_{2} s_{n_{k}, p}^{p}\right)}{\sigma_{k}^{2}}\right\} \\
& +4 \exp \left\{-\frac{\delta^{2} m_{k}\left(2 s_{n_{k}, p}^{2} L_{2} s_{n_{k}, p}^{p}\right)^{1 / 2}}{M_{k} \eta_{k}}\right\},
\end{aligned}
$$

where

$$
\begin{aligned}
& M_{k}=E\left\|\sum_{i=1}^{n_{k}} \epsilon_{i} Z_{i k} I\left\{\left\|Z_{i k}\right\| \leq \eta_{k}\left(2 s_{n_{k}, p}^{2} L_{2} s_{n_{k}, p}^{p}\right)^{1 / 2} / m_{k}\right\}\right\|, \\
& \sigma_{k}^{2}=\sup _{f \in B_{1}^{*}} \sum_{i=1}^{n_{k}} E\left[f^{2}\left(Z_{i k}\right) I\left\{\left\|Z_{i k}\right\| \leq \eta_{k}\left(2 s_{n_{k}, p}^{2} L_{2} s_{n_{k}, p}^{p}\right)^{1 / 2} / m_{k}\right\}\right] .
\end{aligned}
$$

By (3.27) and Levy's inequality, we have

$$
\lim _{k \rightarrow \infty} \frac{M_{k}}{\left.2 s_{n_{k}, p}^{2} L_{2} s_{n_{k}, p}^{p}\right)^{1 / 2}}=0
$$

Thus for $k$ large enough,

$$
\begin{aligned}
& \left.\left[P\left(\left\|\sum_{i=1}^{n_{k}} \epsilon_{i} Z_{i k}\right\|>\epsilon 2 s_{n_{k}, p}^{2} L_{2} s_{n_{k}, p}^{p}\right)^{1 / 2}\right)\right]^{2} \leq C\left\{\left(\frac{1}{2}\right)^{2 m_{k}}+\delta_{k}+\exp \left(-\delta^{2} m_{k}\right)+\exp \left(-\frac{2 \delta^{2} a_{n_{k}}^{2}}{\sigma_{k}^{2}}\right)\right\} \\
& \leq C\left\{\left(\frac{1}{2}\right)^{2 m_{k}}+\delta_{k}+\frac{1}{m_{k}^{2}}+\exp \left(-\frac{2 \delta^{2} a_{n_{k}}^{2}}{\sigma_{k}^{2}}\right)\right\} \leq C\left\{\delta_{k}+\exp \left(-\frac{2 \delta^{2} a_{n_{k}}^{2}}{\sigma_{k}^{2}}\right)\right\}
\end{aligned}
$$

where $C$ is the constant independent of $k$ and varies from line to line, and the second inequality follows from the fact that

$$
\exp (-x) \leq C_{2} \frac{1}{x^{2}} \quad \forall x \geq 1 \text { and for some constant } C_{2}
$$


By (3.30), in order to show (3.35), it is sufficient to prove that

$$
\sum_{k=1}^{\infty} \exp \left(-2 \delta^{2}\left(2 s_{n_{k}, p}^{2} L_{2} s_{n_{k}, p}^{p}\right) / \sigma_{k}^{2}\right)<+\infty
$$

Let $N_{1}=\left\{k \in N, 2 L_{2} s_{n_{k}, p}^{p}<m_{k}=\left[\delta_{k}^{-\frac{1}{2}}\right]\right\}$. For any $k \in N_{i}=1$, we have $\frac{\eta_{k}\left(s_{n_{k}, p}\right.}{\left(2 L_{2} s_{n_{k}, p}^{p}\right)^{1 / 2}}>\frac{\eta_{k}\left(2 s_{n_{k}, p}^{2} L_{2} s_{n_{k}, p}^{p}\right)^{1 / 2}}{m_{k}}$. Thus by the definition of $\left\{Z_{i k}\right\}, M_{k}=0$ and $\sigma_{k}=0$. Hence,

$$
\sum_{k \in N} \exp \left\{-\frac{2 \delta^{2}\left(2 s_{n_{k}, p}^{2} L_{2} s_{n_{k}, p}^{p}\right)}{\sigma_{k}^{2}}\right\}=\sum_{k \in N \backslash N_{1}} \exp \left\{-\frac{2 \delta^{2}\left(2 s_{n_{k}, p}^{2} L_{2} s_{n_{k}, p}^{p}\right)}{\sigma_{k}^{2}}\right\} .
$$

By the argument like the proof of Theorem 2.1, we have that $\sigma_{k} \leq 2^{1+\frac{1}{p}} s_{n_{k}, p}$. Therefore,

$$
\begin{aligned}
& \sum_{k \in N} \exp \left\{-\frac{2 \delta^{2}\left(2 s_{n_{k}, p}^{2} L_{2} s_{n_{k}, p}^{p}\right)}{\sigma_{k}^{2}}\right\}=\sum_{k \in N \backslash N_{1}} \exp \left\{-\frac{2 \delta^{2}}{2^{1+1 / p}} 2 L_{2} s_{n_{k}, p}^{p}\right\} \\
& \leq C \sum_{k \in N \backslash N_{1}} \frac{1}{\left(2 L_{2} s_{n_{k}, p}^{p}\right)^{2}} \leq C \sum_{k \in N \backslash N_{1}} \frac{1}{m_{k}^{2}} \leq C \sum_{k=1}^{\infty} \delta_{k}<+\infty .
\end{aligned}
$$

(3.35) is proved.

Similar to the proof of Theorem 2.1, we easily show that for $\forall \epsilon>0$,

$$
\sum_{k=1}^{\infty} P\left\{\left\|\sum_{i=1}^{n_{k}}\left(Y_{i k}-E Y_{i k}\right)\right\|>\left(2^{1+1 / p}+3 \epsilon\right) a_{n_{k}}\right\}<+\infty
$$

Therefore we have actually proved that

$$
\limsup _{n \rightarrow \infty} \frac{\left\|\sum_{i=1}^{n}\left(X_{i}^{\prime}-E X_{i}^{\prime}\right)\right\|}{a_{n}} \leq 2^{1+1 / p} \quad \text { a.s. }
$$

This completes the proof of Theorem 2.2.

In order to prove Theorem 2.3, we need the following lemma (see Lem. 5 in Einmahl [7]).

Lemma 3.2. Let $\xi: \Omega \rightarrow[0, \infty)$ be a random variable such that

$$
\sum_{n=1}^{\infty} P\left\{\xi>c_{n}\right\}<+\infty
$$

where $\left\{c_{n}\right\}$ is a sequence satisfying for some $\alpha>0$,

$$
c_{n} / n^{\alpha} \text { is nondecreasing. }
$$

Then we have that
(a) $\sum_{n=1}^{\infty} E \xi^{r} I\left\{\xi \leq c_{n}\right\} / c_{n}^{r}<+\infty \quad$ provided that $r>\alpha^{-1}$,
(b) $\sum_{n=1}^{\infty} P\left(\xi>\epsilon c_{n}\right)<+\infty \quad$ for any $\epsilon>0$. 


\section{The proof of Theorem 2.3.}

For each $i \geq 1$, define

$$
X_{i}^{\prime}=X_{i} I\left\{\left\|X_{i}\right\| \leq c_{n}\right\}, \quad X_{i}^{\prime \prime} I\left\{\left\|X_{i}\right\|>c_{i}\right\}=X_{i}-X_{i}^{\prime} .
$$

Then similar to the proof of Theorem 2.2, we easily obtain that

$$
\lim _{n \rightarrow \infty} \sum_{i=1}^{n}\left(X_{i}^{\prime \prime}-E X_{i}^{\prime \prime}\right) / c_{n}=0 \quad \text { a.s. }
$$

and thus, by (2.7) we also have that

$$
\sum_{i=1}^{n}\left(X_{i}^{\prime}-E X_{i}^{\prime}\right) / c_{n} \rightarrow 0 \quad \text { in probability. }
$$

Now by using Lemma 3.2, (2.6) and (2.8) imply that for $r>p=\alpha^{-1}$,

$$
\sum_{n=1}^{\infty} \frac{E\left\|X^{\prime}\right\|^{r}}{c_{n}^{r}}=\sum_{n=1}^{\infty} E\left\|X_{n}\right\|^{r} I\left\{\left\|X_{n}\right\| \leq c_{n}\right\} / c_{n}^{r}=\sum_{n=1}^{\infty} E\|X\|^{r} I\left\{\|X\| \leq c_{n}\right\} / c_{n}^{r}<+\infty .
$$

Hence the conditions in Proposition 3.1 are obtained from (3.37) and (3.38). Therefore, there exists some $\Gamma>0$ such that

$$
\limsup _{n \rightarrow \infty} \frac{\left\|\sum_{i=1}^{n}\left(X_{i}^{\prime}-E X_{i}^{\prime}\right)\right\|}{c_{n}} \leq \Gamma
$$

Again by (3.36), $\limsup _{n \rightarrow \infty}\left\|S_{n}\right\| / c_{n}<\Gamma$. In particular, if $c_{n}=\sigma_{p} n^{1 / p}\left(2 L_{2} n\right)^{1 / 2}$, the conclusion follows from Theorem 2.2.

Finally we give the proof of Theorem 2.3. We first give a proposition which seems to be an extension of Proposition 1.1 in Ledoux and Talagrand [13]. By using Proposition 3.1 in Deng [6] and Theorem 4.12 in Ledoux and Talagrand [14], the proof may follow from the similar argument like the proof of Proposition 1.1 in Ledoux and Talagrand [13] or Theorem 6.17 in Ledoux and Talagrand [14] and thus is omitted.

Proposition 3.3. Let $\left\{X_{i}, n \geq 1\right\}$ be a sequence of independent symmetric B-valued random variables. For any integer $k \geq \omega$ and positive numbers $s$ and $t$, we have that,

$$
\begin{aligned}
& P\left\{\left\|\sum_{i=1}^{N} X_{i}\right\|>t+2 s+8 \omega M\right\} \\
& \leq\left(\frac{K_{0}}{\omega}\right)^{k}+P\left\{\sum_{i=1}^{k}\left\|X_{i}\right\|^{*}>s\right\}+4 \exp \left\{-\frac{t^{q}}{2^{q} q\left(4 \omega \sigma^{p}+24 p M(s / k)^{p-1}\right)^{q / p}}\right\}
\end{aligned}
$$

where $p \in(1,2], q=\frac{p}{p-1}$,

$$
M=E\left\|\sum_{i=1}^{N} u_{i}\right\| \quad \sigma^{p}=\sup _{f \in B_{1}^{*}}\left(\sum_{i=1}^{N} E\left|f\left(X_{i}\right)\right|^{p}\right)
$$

$u_{i}=X_{i} I\left\{\left\|X_{i}\right\| \leq s / k\right\}(i \leq N),\left\{\left\|X_{i}\right\|^{*}, i \leq N\right\}$ is the nondecreasing rearrangement of the sequence $\left\{\left\|X_{i}\right\|, i \leq\right.$ $N\}$, and $K_{0}$ is a universal constant. 


\section{The proof of Theorem 2.4.}

We first prove Theorem 2.4 for $p=1$. In view of $(2.11)$, for $\rho>1$, and each $k$, let $n_{k}=\min \left\{n: s_{n, 1}>\rho^{k}\right\}$. Then,

$$
s_{n_{k}, 1} \sim \rho^{k}, \quad s_{n_{k+1}, 1} / s_{n_{k}, 1} \sim \rho .
$$

Set $I(k)=\left\{n_{k}+1, \ldots, n_{k+1}\right\}$. From Corollary 7.3 in Ledoux and Talagrand [14], it is sufficient to prove that $\limsup _{k \rightarrow \infty}\left\|\sum_{i \in I(k)} X_{i}\right\| / s_{n_{k}, 1}=0$. Now, set $q=\left[2 K_{0}\right]+1, s=\left[2 L_{2} s_{n_{k}, 1}\right]$ and for integer $r$, set $X_{I(k)}^{(r)}=\left\|X_{i}\right\|$ whenever $\left\|X_{i}\right\|$ is the $r$-th maximum of the sample $\left\{\left\|X_{i}\right\|, i \in I(k)\right\}$. On account of (2.12), for all $\epsilon>0$ and for large $k$,

$$
\begin{aligned}
& \sum_{r=1}^{\left[2 L_{2} s_{n_{k}, 1}\right]} X_{I(k)}^{(r)} \leq\left[2 L_{2} s_{n_{k}, 1}\right] \eta_{n_{k}} s_{n_{k}, 1} / L_{2} s_{n_{k}, 1}<\epsilon s_{n_{k}, 1}, \\
& M_{k}=E\left\|\sum_{i \in I(k)} X_{i} I_{\left\{\left\|X_{i}\right\| \leq \epsilon s_{n_{k}, 1} /\left[2 L_{2} s_{n_{k}, 1}\right]\right\}}\right\|=E\left\|\sum_{i \in I(k)} X_{i}\right\|, \\
& \sigma_{k}^{2}=\sup _{f \in B_{1}^{*}}\left(\sum_{i \in I(k)} E f^{2}\left(X_{i} I_{\left\{\left\|X_{i}\right\| \leq \epsilon s_{n_{k}, 1} /\left[2 L_{2} s_{n_{k}, 1}\right]\right\}}\right)\right)=\sup _{f \in B_{1}^{*}}\left(\sum_{i \in I(k)} E f^{2}\left(X_{i}\right)\right) \\
& \quad \leq \sup _{f \in B_{1}^{*}}\left(\sum_{i \in I(k)} E\left|f\left(X_{i}\right)\right| \eta_{i} s_{i, 1} / L_{2} s_{i, 1}\right) \leq s_{n_{k}, 1}^{2} \eta_{n_{k}} / L_{2} s_{n_{k}, 1}
\end{aligned}
$$

and thus for a large $k_{0}$,

$$
\sum_{k \geq k)} \exp \left(-\epsilon^{2} s_{n_{k}, 1}^{2} / \sigma_{k}^{2}\right) \leq \sum_{k \geq k_{0}} \exp \left(-\epsilon L_{2} s_{n_{k}, 1} / \eta_{n_{k}}\right) \leq C \sum_{k \geq k_{o}} \exp \{-2(\log k \log \rho)\}<+\infty .
$$

From Theorem 7.5 in Ledoux and Talagrand [14], it follows that $\limsup _{n \rightarrow \infty} S_{n} / s_{n, 1}=0$.

Next by Borel-Cantelli Lemma and Levy's inequality, in order to establish the theorem for $p \in(1,2)$, it suffices to show that for all $\epsilon>0$,

$$
\sum_{k=1}^{\infty} P\left\{\left\|S_{n_{k}}\right\|>\left(\Lambda_{p}+3 \epsilon\right)\left(2 s_{n_{k}, p}^{q} L_{2} s_{n_{k}, p}^{p}\right)^{1 / q}\right\}<+\infty
$$

where $\left\{n_{k}\right\}$ is the same as above. Taking $\omega=2 K_{0}, k=\left[2 L_{2} s_{n_{k}, p}^{p}\right], s=\epsilon\left(2 s_{n_{k}, p}^{q} L_{2} s_{n_{k}, p}^{p}\right)^{1 / q}, t=\left(\Lambda_{p}+\right.$ $\epsilon)\left(2 s_{n_{k}, p}^{q} L_{2} s_{n_{k}, p}^{p}\right)^{1 / q}$ and applying Proposition 3.3, it is easy to see that

$$
\begin{aligned}
& P\left\{\left\|S_{n_{k}}\right\|>t+2 s+8 \omega M_{k}\right\} \\
& \leq\left(\frac{1}{2}\right)^{\left[2 L_{2} s_{n_{k}, p}^{p}\right]}+P\left(\sum_{i=1}^{\left[2 L_{2} s_{n_{k}, p}^{p}\right]}\left\|X_{i}\right\|^{*}>s\right) \\
& \quad+4 \exp \left(-\frac{\left[(\Lambda+\epsilon)\left(2 s_{n_{k}, p}^{q} L_{2} s_{n_{k}, p}^{p}\right)^{1 / q}\right]^{q}}{2^{q} q\left(4 \omega \sigma_{k}^{p}+24 p M_{k}\left[\epsilon\left(2 s_{n_{k}, p}^{q} L_{2} s_{n_{k}, p}^{p}\right)^{1 / q} /\left(\left[2 L_{2} s_{n_{k}, p}^{p}\right]\right)\right]^{p-1}\right)^{q / p}}\right)
\end{aligned}
$$


where

$$
\begin{aligned}
& M_{k}=E\left\|\sum_{i=1}^{n_{k}} X_{i} I_{\left\{\left\|X_{i}\right\| \leq s / k\right\}}\right\|, \\
& \sigma_{k}^{p}=\sup _{f \in B_{1}^{*}}\left(\sum_{i=1}^{n_{k}} E\left|f\left(X_{i}\right) I_{\left\{\left\|X_{i}\right\| \leq s / k\right\}}\right|^{p}\right)
\end{aligned}
$$

and thus $M_{k}=\left\|S_{n_{k}}\right\|, \sigma_{k}^{p}=s_{n_{k}, p}^{p}$ for sufficiently large $k$ since $\left\|X_{i}\right\| \leq \eta_{i} s_{i, p} /\left(L_{2} s_{i, p}^{p}\right)^{1 / p} \leq \epsilon\left(2 s_{n_{k}, p}^{q} L_{2} s_{n_{k}, p}^{p}\right)^{1 / q} /$ $\left(2 L_{2} s_{n_{k}, p}^{p}\right) \leq s / k$. Again, by using the standard method, it is easy to see that $(2.13)$ implies that $\lim _{n \rightarrow \infty} \alpha_{k} \equiv$ $\lim _{n \rightarrow \infty} E|| S_{n} \| /\left(2 s_{n, p}^{q} L_{2} s_{n, p}^{p}\right)^{1 / q}=0$. Now note that for sufficiently large $k$,

$$
\begin{aligned}
& \quad \sum_{i=1}^{\left[2 L_{2} s_{n_{k}, p}^{p}\right]}\left\|X_{i}\right\|^{*} \leq\left[2 L_{2} s_{n_{k}, p}^{p}\right] \eta_{n_{k}} s_{n_{k}, p} /\left(L_{2} s_{n_{k}, p}^{p}\right)^{1 / p} \leq s, \\
& 24 p M_{k}\left[\epsilon\left(2 s_{n_{k}, p}^{q} L_{2} s_{n_{k}, p}^{p}\right)^{1 / q} /\left(\left[2 L_{2} s_{n_{k}, p}^{p}\right]\right)\right]^{p-1} \\
& \leq 24 p \epsilon^{p-1} M_{k} s_{n_{k}, p}^{p-1} /\left(2 L_{2} s_{n_{k}, p}^{p}\right)^{1 / q} \\
& \leq 24 p \epsilon^{p-1} s_{n_{k}, p}^{p}\left\|S_{n_{k}}\right\| /\left(2 s_{n_{k}, p}^{q} L_{2} s_{n_{k}, p}\right)^{1 / q} \leq C \alpha_{k} s_{n_{k}, p}^{p} .
\end{aligned}
$$

Therefore, for $k$ large enough,

$$
\begin{aligned}
& P\left\{\left\|S_{n_{k}}\right\|>\left(\Lambda_{p}+3 \epsilon\right) 2 s_{n_{k}, p}\left(L_{2} s_{n_{k}, p}^{p}\right)^{1 / q}\right\} \\
& \leq P\left\{\left\|S_{n_{k}, p}\right\|>t+2 s+8 \omega M_{k}\right\} \\
& \leq\left(\frac{1}{2}\right)^{2 \log _{2} \rho^{p k}}+4 \exp \left\{-\frac{\left[\left(16 K_{0}\right)^{1 / p} q^{1 / q}+\epsilon\right]^{q} 2 s_{n_{k}, p}^{q} L_{2} s_{n_{k}, p}^{p}}{2^{q} q\left(4 \omega+\alpha_{k}\right) s_{n_{k}, p}^{q}}\right\} \\
& \leq 2^{-2 \log (p k \log \rho)}+4 \exp \left\{-\frac{\left[\left(8 K_{0}\right)^{1 / p} q^{1 / q}+\epsilon / 2^{1 / p}\right]}{q\left(8 K_{0}+\alpha_{k}\right)^{q / p}} \log _{2} s_{n_{k}, p}^{p}\right\} \\
& \leq 2^{-2 \log (p k \log \rho)}+4 \exp \left\{-\left(1+\frac{\epsilon}{2}\right) \log _{2} s_{n_{k}, p}^{p}\right\},
\end{aligned}
$$

from which, (3.10) follows. For $p=2$ the result follows from Kolmogorov's LIL (see Chen [4]). The proof of Theorem 2.4 is complete.

Acknowledgements.

This work is partly supported by a grant from the Natural Science and Engineering Research Council of Canada.

\section{REFERENCES}

[1] A. de Acosta, Inequalities for $B$-valued random variables with application to the law of large numbers. Ann. Probab. 9 (1981) $157-161$.

[2] B. von Bahr and C. Esseen, Inequalities for the $r$ th absolute moments of a sum of random variables, $1 \leq r \leq 2$. Ann. math. Statist. 36 (1965) 299-303.

[3] X. Chen, On the law of iterated logarithm for independent Banach space valued random variables. Ann. Probab. 21 (1993) $1991-2011$.

[4] X. Chen, The Kolmogorov's LIL of B-valued random elements and empirical processes. Acta Mathematica Sinica 36 (1993) $600-619$.

[5] Y.S. Chow and H. Teicher, Probability Theory: Independence, Interchangeability, Martigales. Springer-Verlag, New York (1978).

[6] D. Deng, On the Self-normalized Bounded Laws of Iterated Logarithm in Banach Space. Stat. Prob. Lett. 19 (2003) $277-286$. 
[7] U. Einmahl, Toward a general law of the iterated logarithm in Banach space. Ann. Probab. 21 (1993) $2012-2045$.

[8] E. Gine and J. Zinn, Some limit theorem for emperical processes. Ann. Probab. 12 (1984) 929-989.

[9] A. Godbole, Self-normalized bounded laws of the iterated logarithm in Banach spaces, in Probability in Banach Spaces 8, R. Dudley, M. Hahn and J. Kuelbs Eds. Birkhäuser Progr. Probab. 30 (1992) 292-303.

[10] P. Griffin and J. Kuelbs, Self-normalized laws of the iterated logarithm. Ann. Probab. 17 (1989) $1571-1601$.

[11] P. Griffin and J. Kuelbs, Some extensions of the LIL via self-normalizations. Ann. Probab. 19 (1991) $380-395$.

[12] M. Ledoux and M. Talagrand, Characterization of the law of the iterated logarithm in Babach spaces. Ann. Probab. 16 (1988) $1242-1264$.

[13] M. Ledoux and M. Talagrand, Some applications of isoperimetric methods to strong limit theorems for sums of independent random variables. Ann. Probab. 18 (1990) 754-789.

[14] M. Ledoux and M. Talagrand, Probability in Banach Space. Springer-Verlag, Berlin (1991).

[15] R. Wittmann, A general law of iterated logarithm. Z. Wahrsch. verw. Gebiete 68 (1985) 521-543. 